\title{
Um modelo de jogador baseado em Estilos de Aprendizagem
}

\author{
Diego Lopes Marques', Danielle Rousy ${ }^{1}$, Carla Silva ${ }^{2}$ \\ ${ }^{1}$ Programa de Pós Graduação em Informática (PPGI) \\ Centro de Informática / Universidade Federal da Paraíba (CI/UFPB) \\ João Pessoa - PB - Brasil \\ ${ }^{2}$ CIn/UFPE Av. Jornalista Aníbal Fernandes 50740-560 \\ Recife - PE - Brasil \\ diego_silva@ppgi.ufpb.br, danielle@ci.ufpb.br, ctlls@cin.ufpb.br
}

\begin{abstract}
Each individual has a different profile that influences the way it organizes and processes information. This profile can be described as learning style where every person learns a particular subject or content according to their individual differences, thus contributing to the construction of the teaching / learning process. This is one of the reasons why educational games can be so powerful in this process, since the students can learn playing and consequently joining to it, the game adaptation according to your profile, increasing the learning. This article proposes a player model based on learning styles for educational games.

Resumo. Cada indivíduo possui um perfil diferente que influencia na forma de como ele organiza e processa informação. Esse perfil pode ser descrito como estilo de aprendizagem onde cada pessoa aprende um determinado assunto ou conteúdo de acordo com suas diferenças individuais, contribuindo assim para a construção do processo de ensino/aprendizagem. Esse é um dos motivos pelo qual os jogos educativos podem ser tão poderosos nesse processo, uma vez que os alunos podem aprender brincando e consequentemente unindo a isso a adaptação do jogo de acordo com o seu perfil potencializando à aprendizagem. Este artigo propõe um modelo de jogador baseado em estilos de aprendizagem para jogos educativos.
\end{abstract}

\section{Introdução}

O processo de ensino e aprendizagem passou a contar com a colaboração da tecnologia e isso tem contribuído diretamente no conceito chamado de aprender a aprender, onde o aluno é autônomo em relação ao seu aprendizado (Batista, 2014). Isso tem se potencializado pela utilização de jogos na educação,pois geram nos jogadores a predisposição para aprender, uma vez que são criadas situações de desafios (Moita, 2007). Além disso, os jogos educativos costumam fornecer feedback ao aluno com o objetivo de modificar o seu pensamento e/ou o seu comportamento, promovendo a aprendizagem e aumentando o seu conhecimento, suas habilidades e sua compreensão em relação a um determinado conteúdo (Shute, 2008).

Entretanto, cada usuário processa e absorve informações de maneira particular, ou seja, cada pessoa possui um perfil único de aprender, também conhecido como 
estilo. De acordo com Felder e Brent (2005), estilos de aprendizagem são processos cognitivos utilizados para aprender algo durante a vida. Além disso, os estilos de aprendizagem têm sido utilizados e reconhecidos como sendo um fator humano importante e que afeta diretamente o desempenho de aprendizagem de cada aluno (Amaral e Barros, 2007; Scaico, 2012).

Aliado a isso, uma das abordagens que podem ser utilizadas para o reconhecimento dos estilos de aprendizagem em jogos digitais, é o de modelo de jogador. Machado (2011) retrata que modelo de jogador consiste em modelar características e comportamentos do aluno durante o jogo, a fim de melhorar alguns aspectos do jogo, como por exemplo, os feedbacks que o jogo pode fornecer ao usuário.

Diante da necessidade de se considerar os estilos de aprendizagem de cada aluno no processo de ensino e aprendizagem mediado por jogos educacionais, este trabalho tem como objetivo definir um modelo de jogador baseado em estilos de aprendizagem que permita fornecer feedbacks adaptativos no jogo. Esse modelo foi implementado em um jogo de perguntas e respostas, denominado MyQuímica (Lopes et al, 2012).

\section{Estilos de Aprendizagem}

De acordo com Felder e Brent (2005), as teorias que envolvem estilos de aprendizagem contribui para a construção do processo de ensino aprendizagem do aluno, e tem como objetivo aumentar a capacidade deles, fazendo com que a aprendizagem seja um ato motivador, comum e cotidiano.

A partir de várias pesquisas, Felder e Silverman (1988) desenvolveram um modelo de estilos de aprendizagem baseado em dimensões, que trabalham com extremidades opostas, sendo elas: visual/verbal, sensorial/intuitivo, ativo/reflexivo, e sequencial/global. A tabela 1 descreve cada um desses estilos.

Tabela 1. Descrição dos estilos de aprendizagem proposto por Felder e Silverman (1988)

\begin{tabular}{|l|l|}
\hline Estilo & Definição \\
\hline Ativo & $\begin{array}{l}\text { Processam informação fazendo alguma atividade, ou sejam testando o } \\
\text { conteúdo }\end{array}$ \\
\hline Reflexivo & $\begin{array}{l}\text { Tendem a processar primeiramente a informação introspectivamente, } \\
\text { pensando muito nas coisas antes de testá-las }\end{array}$ \\
\hline Visual & $\begin{array}{l}\text { Obtêm informações preferencialmente a partir de imagens visuais como, } \\
\text { figuras, diagramas, gráficos e esquemas }\end{array}$ \\
\hline Verbal & $\begin{array}{l}\text { Obtêm informação através daquilo que lêem e ouvem (ainda mais } \\
\text { daquilo que ouvem e repetem) }\end{array}$ \\
\hline Sensorial & $\begin{array}{l}\text { Prestam atenção a detalhes e não gostam de conceitos abstratos, e } \\
\text { preferem que o conteúdo esteja relacionado com o mundo real }\end{array}$ \\
\hline Intuitivo & $\begin{array}{l}\text { Lidam bem com abstrações e ficam entediados com os detalhes, e } \\
\text { preferem problemas que exigem raciocínio inovador. }\end{array}$ \\
\hline
\end{tabular}


V Congresso Brasileiro de Informática na Educação (CBIE 2016)

Anais do XXII Workshop de Informática na Escola (WIE 2016)

\begin{tabular}{|l|l|}
\hline Sequencial & $\begin{array}{l}\text { Absorvem informações na medida em que ela é apresentada, de forma } \\
\text { linear }\end{array}$ \\
\hline Global & $\begin{array}{l}\text { Têm que ter um conhecimento mais completo do conteúdo, ou seja, de } \\
\text { maneira mais abrangente }\end{array}$ \\
\hline
\end{tabular}

Os autores também propõem um questionário, denominado Índice de Estilo de Aprendizagem (ILS), que tem como finalidade descobrir o estilo de aprendizagem do aluno, a partir da resolução de algumas perguntas.

\section{Trabalhos Relacionados}

Encontramos alguns trabalhos correlacionados com o foco da pesquisa aqui apresentada. Um deles é o trabalho de Soflano, Connolly e Hainer (2015). Este trabalho apresenta um jogo de perguntas e respostas, com o objetivo de ensinar a linguagem estruturada de consulta a banco de dados, SQL ${ }^{1}$. O trabalho descreve um estudo empírico que investiga a eficácia da utilização de diferentes grupos de alunos com estilos de aprendizagem distintos, uma vez que o jogo se adapta ao perfil de cada jogador, identificado a partir do questionário ILS.

O trabalho de Andersen (2013) apresenta 3 (três) jogos, com finalidade de identificar o perfil do aluno, e verificar a influência da aprendizagem. O objetivo era identificar a preferência dos usuários, através do questionário ILS, que era respondido no próprio jogo, antes dos usuários começarem a tentar resolver os desafios. E a partir da análise dos dados registrados no jogo verificarem as diferenças do "jogar" de cada estilo de aprendizagem analisado na pesquisa.

Com base nos estudos realizados, nossa proposta é definir um modelo de jogador baseado em estilos de aprendizagem que possa ser usado em um jogo educacional, do tipo pergunta e resposta, para fornecer um feedback personalizado ao estilo de aprendizagem do jogador. Assim, o modelo de jogador teria como um dos seus componentes o estilo de aprendizagem do jogador. Essa proposta é apresentada na próxima seção.

\section{O Modelo}

O modelo de jogador que resultou neste trabalho foi concebido da seguinte forma: $\mathrm{O}$ jogador responde o questionário ILS para identificar o seu estilo de aprendizagem, antes de começar a solucionar os desafios propostos pelo jogo. Cada desafio possui características como: níveis, respostas, sons, imagens, dicas, pontuação e questões adicionais. Essas características são arquivadas no jogo, por meio de um banco de dados, de acordo com o desempenho do jogador, que unindo ao seu estilo identificado, compõem o modelo de jogador proposto. A figura 1 apresenta este modelo.

\footnotetext{
${ }^{1}$ Do inglês Structured Query Language
} 


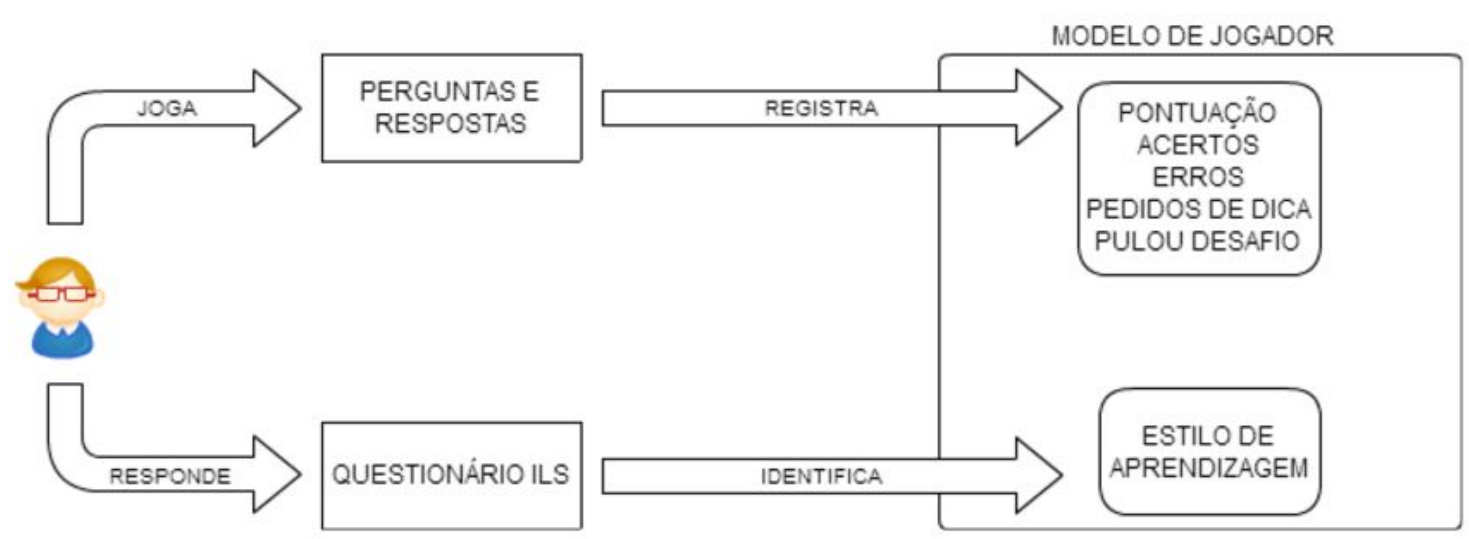

Figura 1. Modelo de jogador

\subsection{Validação do Modelo}

Para validar a proposta descrita nesse trabalho, o modelo foi implementado no jogo MyQuímica, um jogo de pergunta e resposta que tem como finalidade auxiliar os alunos no aprendizado das nomenclaturas químicas, bem como na familiarização dos mesmos com a tabela periódica.

Ele se destina a alunos do Ensino Médio que já estudaram o conteúdo de nomenclaturas químicas e desejam exercitá-lo de uma maneira diferente. O jogo propõe aos alunos vários desafios que consistem na descoberta de fórmulas de compostos químicos cuja nomenclatura é mostrada. Um vídeo explicativo do jogo pode ser visualizado no YouTube ${ }^{2}$. O MyQuímica também grava log dos dados da sessão do jogo de cada usuário. Dessa forma, o sistema registra todos os passos realizados pelos jogadores no jogo, desde apertar em algum botão, até solucionar um desafio.

\subsection{Implementação do Modelo}

A implementação foi desenvolvida em um período de 3 (três) meses, e se baseou nos seguintes aspectos:

- Verificação da implementação atual do jogo

- Refatoração do código para implementação do modelo de jogador

- Implementação do modelo de jogador

- Testes com a versão implementada

- Geração de versão implementada para a avaliação.

Na primeira etapa que envolveu a verificação da implementação atual do jogo, foi constatado que era necessário fazer uma reformulação nos códigos do MyQuímica. Isso pois o jogo não estava habilitado para receber as informações do perfil do jogador. Dessa forma, foi feita uma breve análise sobre quais classes do código fonte e quais tabelas do banco de dados sofreriam alterações. Uma vez verificado o estado atual do

\footnotetext{
${ }^{2} \mathrm{https}: / /$ www.youtube.com/watch?v=tFQWS4Gpo5c
} 
V Congresso Brasileiro de Informática na Educação (CBIE 2016)

Anais do XXII Workshop de Informática na Escola (WIE 2016)

jogo MyQuímica, foi feita a refatoração do código para a implementação do modelo de jogador.

Após a refatoração do código, foi então implementado a nova versão do jogo MyQuímica, de acordo com as novas classes e tabelas de banco de dados. Com isso, foram realizados alguns testes para saber se a implementação tinha sido concluída, sem maiores problemas no que diz respeito a disponibilização do jogo para os usuários realizarem a avaliação Por fim, foi gerada uma versão do jogo com as novas implementação.

\section{Refinamento e Verificação do Modelo}

\subsection{Implementação do Modelo}

Foi realizado um primeiro semi-experimento que contemplou o refinamento do modelo através da aplicação de uma versão do jogo MyQuímica que não possuía personalização de feedback com alguns alunos do Ensino Médio, a fim de identificar elementos de customização conforme, o estilo de aprendizagem do aluno.

O público alvo foi de alunos do $1^{\circ}$ e $2^{\circ}$ ano do ensino médio de escolas públicas, que já tiveram algum contato com a disciplina de química e com o assunto de nomenclaturas químicas. Considerando esse público, tivemos a participação de 27 alunos, sendo 16 (dezesseis) do $1^{\circ}$ ano do ensino médio, e 11 do $2^{\circ}$ ano do ensino médio.

A pesquisa se deu da seguinte forma: de maneira voluntária, 1 (um) aluno por vez se dirigia até o local para a realização da avaliação. Existia um mediador portando um tablet, o qual era entregue ao aluno que jogava entre 10 e 20 minutos, até que desistisse de jogar ou que realizasse todos os desafios propostos. Antes de começar a responder os desafios propostos pelo jogo, os participantes respondiam um questionário que foi implementado no próprio jogo, para identificar o seu estilo de aprendizagem, e o seu comportamento. Esse questionário criado por Felder e Silverman (1988), adaptado por Vieira Jr (2012), e que classifica os estilos em: em: ativos /reflexivos; sensoriais/intuitivos; visuais/verbais; e sequenciais/globais.

Após a finalização da utilização do jogo por todos os participantes da pesquisa, fez-se uma entrevista com perguntas baseadas na experiência que os alunos tiveram com o jogo. Essas perguntas serviram para colher informações e características adicionais que pudessem ajudar no refinamento do modelo e identificar de maneira mais efetiva em que o feedback poderia ser diferenciado conforme o estilo de aprendizagem. Além disso, serviu para identificar informações que não foram medidas através do questionário, nem na resolução dos desafios do jogo, tais como: pontuação, tempo de resolução, acertos e erros dos desafios, quantidade de pedidos de dicas e quantidade de vezes que o aluno "pulou" o desafio. Essas informações foram definidas como um conjunto de elementos que ajudaram a selecionar alguns pontos de personalização do feedback para o usuário e serviram para ajudar a manter a adaptação com o estilo de aprendizagem de cada jogador.

As perguntas realizadas durante essa entrevista foram: teve alguma dificuldade na resolução dos desafios? As dicas dadas pelo jogo o ajudaram na resolução dos 
V Congresso Brasileiro de Informática na Educação (CBIE 2016)

Anais do XXII Workshop de Informática na Escola (WIE 2016)

desafios? Que pontos negativos e positivos você observou e que sugestões você pode nos dar para melhoria do jogo?

Da análise dos dados das entrevistas identificamos alguns pontos de diferenciação de elementos de jogo por estilo de aprendizagem que poderiam ser utilizados para personalizar o feedback, são eles: sons, destaques (highlights), questão complementar, vídeos/animações, pontuação final, respostas certas e erradas, tempo e níveis. Além disso, outras respostas que não se enquadraram nas categorias anteriores, foram definidas como Outros. A tabela 2 apresenta os elementos identificados de acordo com cada perfil.

Tabela 2. Elementos identificados de acordo com cada estilo de aprendizagem, a partir da entrevista realizada com os alunos

\begin{tabular}{|l|c|c|c|c|c|c|c|c|}
\hline Elementos & Ativo & Reflexivo & Sensorial & Intuitivo & Visual & Verbal & Sequencial & Global \\
\hline Sons & $\mathrm{X}$ & & & $\mathrm{X}$ & $\mathrm{X}$ & $\mathrm{X}$ & & \\
\hline Destaque na Tabela & & & & & $\mathrm{X}$ & & & $\mathrm{X}$ \\
\hline Questão complementar & & & $\mathrm{X}$ & $\mathrm{X}$ & & & & \\
\hline Vídeos/Animações & $\mathrm{X}$ & & & & $\mathrm{X}$ & & $\mathrm{X}$ & $\mathrm{X}$ \\
\hline Pontuação Final & & $\mathrm{X}$ & $\mathrm{X}$ & & & & & $\mathrm{X}$ \\
\hline Erros e Acertos & & $\mathrm{X}$ & & $\mathrm{X}$ & & & & \\
\hline $\begin{array}{l}\text { Tempo para não deixar } \\
\text { pedir dica }\end{array}$ & $\mathrm{x}$ & $\mathrm{X}$ & & & & & & \\
\hline Niveis & & & $\mathrm{X}$ & & & & $\mathrm{X}$ & \\
\hline Outros & & & & & & $\mathrm{X}$ & & \\
\hline
\end{tabular}

Dos 9 (nove) elementos identificados, foram implementados 8 (oito). O elemento videos/animações não foi implementado por conta de limitações de tempo. Já o elemento outros, por serem diversos, não entraram na implementação do modelo. Portanto, através da identificação desses elementos, o refinamento serviu para extrair de forma mais precisa o que poderia ser personalizado no feedback conforme o estilo de aprendizagem assumido pelo aluno e, a partir desse modelo mais refinado, o jogo foi novamente utilizado pelos alunos, ou seja, foi feita uma verificação do modelo implementado. A figura 2 apresenta o modelo refinado. 
V Congresso Brasileiro de Informática na Educação (CBIE 2016)

Anais do XXII Workshop de Informática na Escola (WIE 2016)

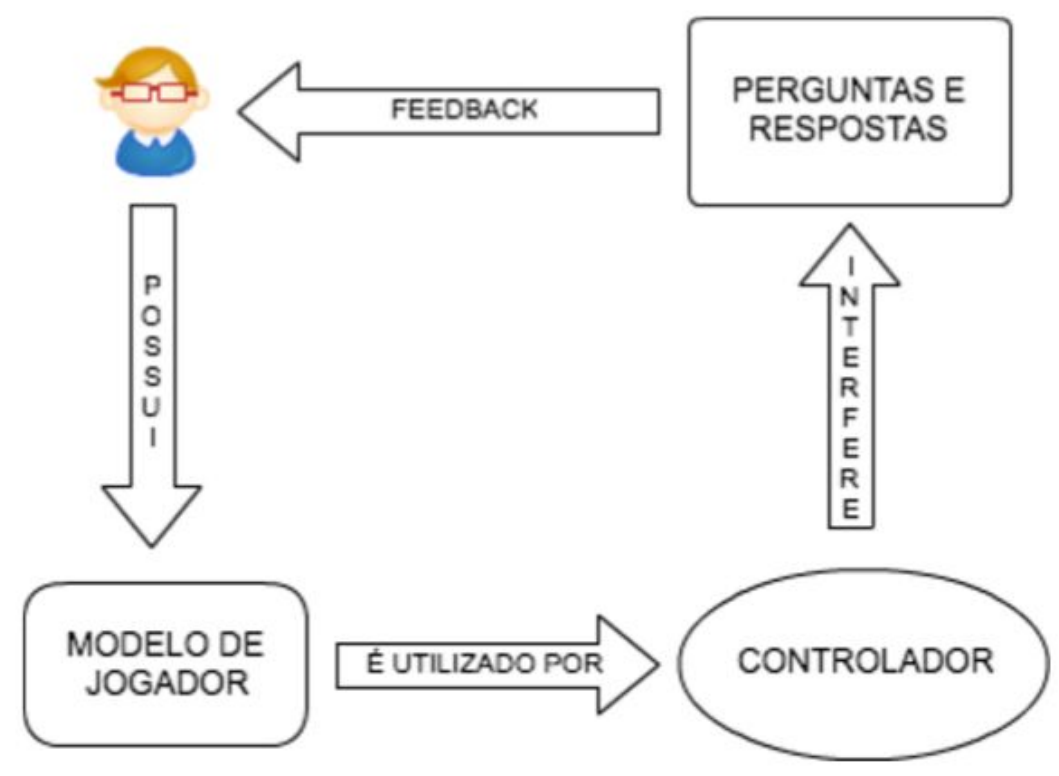

Figura 2. Modelo de jogador após o refinamento.

\subsection{Verificação do Modelo}

Para verificar se a versão adaptativa do jogo, influenciou de alguma forma no ensino/aprendizagem dos alunos, foi de acordo com os feedbacks implementados baseado nos elementos descritos na tabela 1 , foi realizado outro semi-experimento. A Figura 3 apresenta um feedback fornecido ao jogador com um estilo visual a partir da versão não adaptativa, onde o agente pedagógico da aplicação, representado por um químico, apresenta um balão de diálogo informando em quais colunas da tabela periódica se encontram os elementos que compõem o desafio proposto. Já a Figura 4 apresenta um feedback adaptativo também ao estilo de jogador visual. Entretanto, essa figura apresenta um feedback que teve como elementos apresentados na Tabela 2. Na ocasião, o elemento foi o de destaque da tabela, onde as colunas que contêm os elementos para a resolução do desafio ficam com um destaque (highlight) maior em relação às demais. 
V Congresso Brasileiro de Informática na Educação (CBIE 2016)

Anais do XXII Workshop de Informática na Escola (WIE 2016)

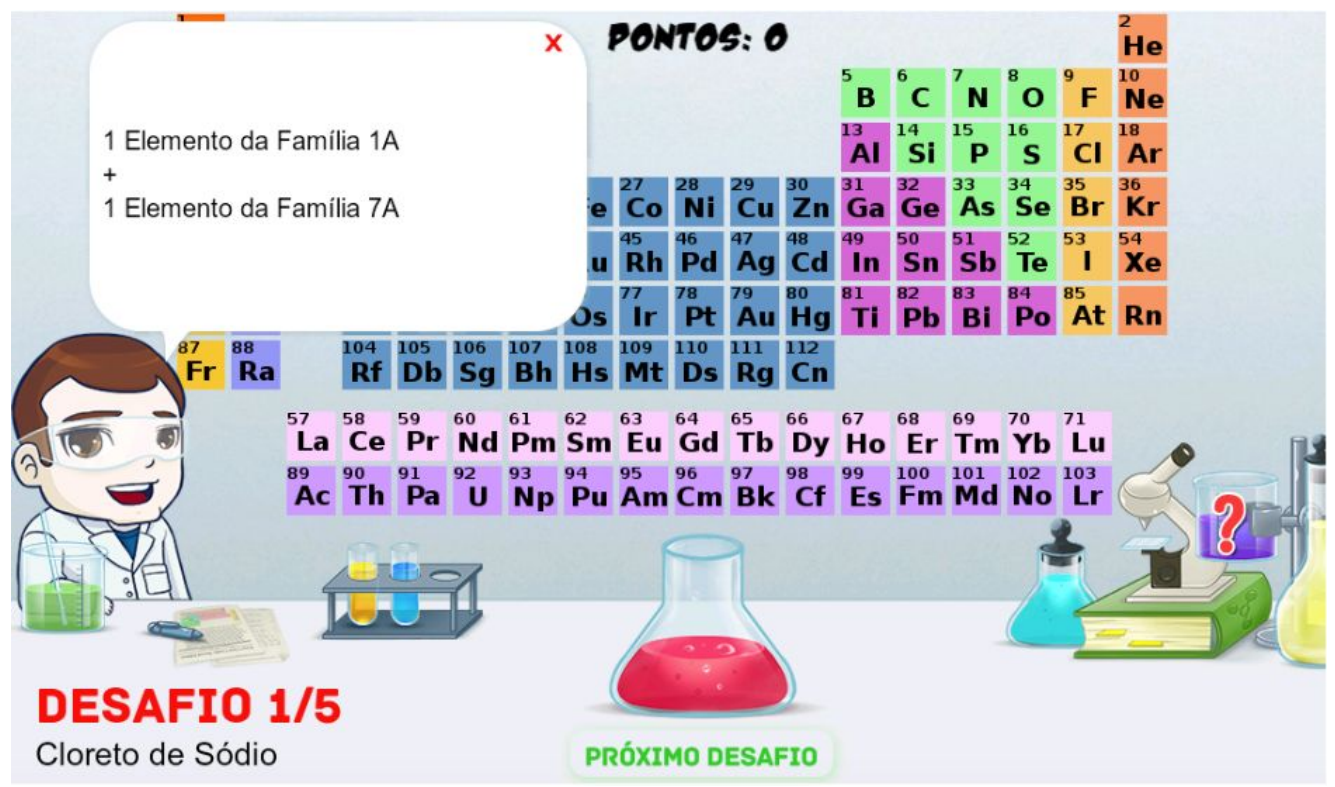

Figura 3. Feedback ao usuário com estilo de aprendizagem visual na versão do jogo não adaptativa.

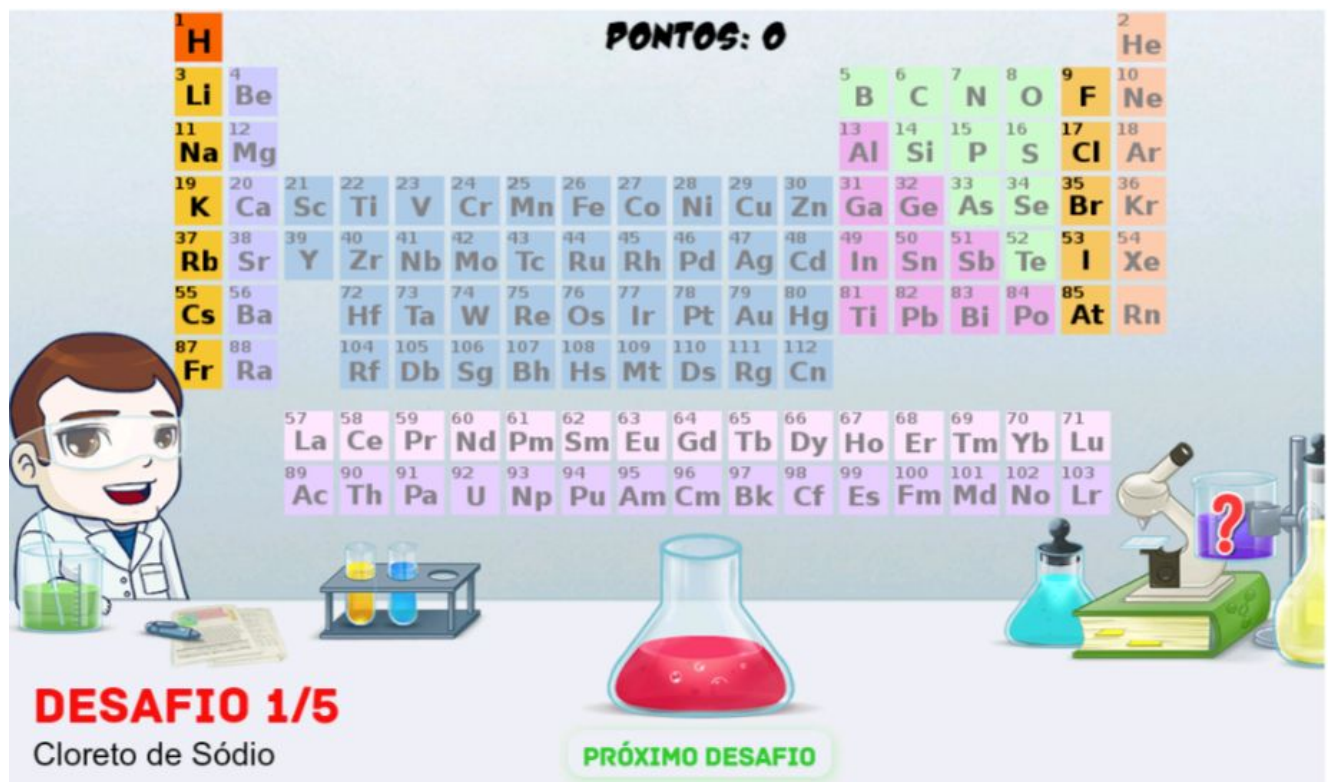

Figura 4. Feedback ao usuário com estilo de aprendizagem visual na versão do jogo adaptativa

\section{Conclusões e Trabalhos Futuros}

Considerando as variáveis investigadas neste trabalho, conclui-se que na opinião dos alunos que participaram dos experimentos aqui apresentados, o jogo adaptativo apresentou melhores resultados do que a versão do jogo não adaptativo. Além disso, a variação dos feedbacks apresentados, de acordo com cada estilo de aprendizagem identificado, mostraram indícios que os alunos se motivaram mais na resolução dos desafios, bem como um aumento nos resultados em termos de pontuação. Contudo, é 
V Congresso Brasileiro de Informática na Educação (CBIE 2016)

Anais do XXII Workshop de Informática na Escola (WIE 2016)

necessário que se tenha um período maior para realizar um novo experimento, e com isso afirmar com mais certeza os resultados identificados ao longo deste trabalho.

Como trabalhos futuros planeja-se evoluir o jogo considerando os pontos negativos e sugestões resultantes das entrevistas realizadas com os alunos após a utilização do jogo. Além disso, pretende-se submeter futuras versões do jogo a avaliações mais amplas, com mais alunos, uma vez que pela limitação do tempo e também por estar próximo do período de férias dos alunos, foi difícil encontrar voluntários que pudessem participar do experimento.

\section{Referências}

Andersen, S. T. “The Learning Style Game”, Master Thesis, Aalborg Universitet, 2013.

Amaral, S. F and Barros, D. M. V. (2007) "Estilos de Aprendizagem no contexto educativo de uso das tecnologias digitais interativas" Disponível em: $<$ http://www.lantec.fe.unicamp.br/ lantec/pt/tvdi_portugues/daniela.pdf. $>$ Consultado: 18 de Mar. 2016.

Batista, E. J. S., Silva, J. B., Primo, W. M., and Silva, R. S. (2014). Desenvolvimento de um aplicativo para android com questões do poscomp como um objeto de aprendizagem para o auxílio no ingresso a programas de pos-graduação. V Escola Regional de Informática de MS.

Dias, George Paulus Pereira. Estilo de aprendizagem Ativo- Reflexivo e jogo de empresas: (des) entrosamento para o aprendizado de planejamento e controle da produção. 2014. Tese (Doutorado em Engenharia de Produção) - Escola Politécnica, Universidade de São Paulo, São Paulo, 2014.

Felder, R., Brent, R. (2005).: Understanding Student Differences. J. Engr. Education, 94 (1), 57-72.

Felder, R. M.; Silverman, L. K. (1988). Learning and Teaching Styles in Engineering Education. Engineering Education, 1988, vol. 78, n.7, April, p. 674-681.

Lopes, D. ; Dantas, A. ; Scaico, P. D.: . Exercitando Nomenclaturas Químicas com Dispositivos Móveis: Uma experiência prática com o uso do MyQuímica. In: Anais do VII LACLO - Confêrencia Latinoamericana de Objetos e Tecnologias de Aprendizagem, 2012

Machado, M. (2011). "Player modeling: Towards a common taxonomy". In: COMPUTER GAMES (CGAMES), 2011 16TH INTERNATIONAL CONFERENCE ON.

Moita, F. (2007) Game On: jogos eletrônicos na vida da geração @. Campinas: Alínea.

Scaico, P.; Silva, D. L. M. ; Silva, M. A. A. ; Cassia, J. ; Vieira, S. ; Falcao, E. S. F. . Implementação de um Jogo Sério para o Ensino de Programação para Alunos do Ensino Médio Baseado em mlearning. In: CSBC 2012 - WEI, 2012, Curitiba. Anais do XX Workshop sobre Educação em Computação, 2012.

Shute, V. J. (2008) "Focus on formative feedback". Review of Educational Research, 78(1), 153-189. 
V Congresso Brasileiro de Informática na Educação (CBIE 2016)

Anais do XXII Workshop de Informática na Escola (WIE 2016)

Soflano, M.; Connolly, T. M.; Hainer, T. (2015) "An application of adaptive games-based learning based on learning style to teach SQL". Journal of Research on Computers \& Education. Vol 86. Pages 192-211. August 2015

Vieira Jr.: Planejamento de um ambiente virtual de aprendizagem baseado em interfaces dinâmicas e uma aplicação ao estudo de potência elétrica. 233 f. Tese (Doutorado) Universidade Estadual Paulista "Júlio Mesquita Filho". Campus de Ilha Solteira, 2012. 